\title{
Applications of Ultrasonic Techniques in Oil and Gas Pipeline Industries: A Review
}

\author{
Wissam M. Alobaidi1 ${ }^{*}$, Entidhar A. Alkuam², Hussain M. Al-Rizzo1, Eric Sandgren1 \\ ${ }^{1}$ Systems Engineering Department, Donaghey College of Engineering \& Information Technology, University of \\ Arkansas at Little Rock, Little Rock, Arkansas, USA \\ ${ }^{2}$ Department of Physics and Astronomy, College of Arts, Letters, and Sciences, University of Arkansas at Little \\ Rock, Little Rock, Arkansas, USA \\ Email: "wmalobaidi@ualr.edu
}

Received 8 May 2015; accepted 3 July 2015; published 6 July 2015

Copyright @ 2015 by authors and Scientific Research Publishing Inc. This work is licensed under the Creative Commons Attribution International License (CC BY). http://creativecommons.org/licenses/by/4.0/

(c) (i) Open Access

\section{Abstract}

The diversity of ultrasound techniques used in oil and gas pipeline plants provides us with a wealth of information on how to exploit this technology when combined with other techniques, in order to improve the quality of analysis. The fundamental theory of ultrasonic nondestructive evaluation (NDE) technology is offered, along with practical limitations as related to two factors (wave types and transducers). The focus is limited to the two main techniques used in pipe plants: First, straight beam evaluation and second, angle beam evaluation. The depth of defect (DD) is calculated using straight beam ultrasonic in six different materials according to their relative longitudinal wave $(L W)$ velocities. The materials and respective velocities of $L W$ are: rolled aluminum $(6420 \mathrm{~m} / \mathrm{s})$, mild steel $(5960 \mathrm{~m} / \mathrm{s})$, stainless steel-347 $(5790 \mathrm{~m} / \mathrm{s})$, rolled copper $(5010 \mathrm{~m} / \mathrm{s})$, annealed copper $(4760 \mathrm{~m} / \mathrm{s})$, and brass $(4700 \mathrm{~m} / \mathrm{s})$. In each material eight defects are modeled; the first represents $100 \%$ of the material thickness $(D), 50.8 \mathrm{~mm}$. The other seven cases represent the $D D$, as $87.5 \%$ of the material thickness, $75 \%, 62.5 \%, 50 \%, 37.5 \%, 25 \%$, and $12.5 \%$, respectively. Using angle beam evaluation, several parameters are calculated for six different reflection angles $\left(\beta_{\mathrm{R}}\right)\left(45^{\circ}, 50^{\circ}, 55^{\circ}, 60^{\circ}, 65^{\circ}\right.$ and $\left.70^{\circ}\right)$. The surface distance (SD), $1 / 2$ skip distance (SKD), full SKD, and $11 / 2$ SKD, $1 / 2$ sound path (SP) length, full SP, and $1 \frac{1 / 2}{2}$ SP are calculated for each $\beta_{R}$. The relationship of SKD and SP to the $\beta_{R}$ is graphed. A chief limitation is noted that ultrasound testing is heavily dependent on the expertise of the operator, and because the reading of the outcome is subjective, precision may be hard to achieve. This review also clarifies and discusses the options used in solving the industrial engineering problem, with a comprehensive historical summary of the information available in the literature. Merging various NDE inspection techniques into the testing of objects is discussed. Eventually, it is hoped to find a suitable technique combined with ultrasonic inspection to deliver highly effective remote testing.

\footnotetext{
${ }^{*}$ Corresponding author.
} 


\section{Keywords}

\section{Ultrasonic Testing, Guided Waves, Nondestructive Testing (NDT), Pipe Inspection, Pipe Thickness Measurement}

\section{Introduction}

Ultrasonic testing is one of the important techniques of nondestructive testing (NDT). It uses ultra-high-frequency sonic energy to locate and identify discontinuities in materials that are both on and below the surface of the material (such as metals or plastic, commonly used to make pipes, depending on the application) [1]-[5].

In 2007 D. S. Caravaca et al. [6] studied polyethylene pipe joints and detection of improper preparation of the joints using a phased array technique (PAT). The problems that arise with the electrofusion type of bonding used for the pipe are analogous to those that occur in metal welds for steel pipe. This paper aims to offer an ultrasonic method for evaluating polyethylene pipe welds, in pipeline used for gas and water distribution, along with results from both laboratory and field experiments [6].

The sonic energy passes through the substrate. There is a reduction in energy intensity, as well as reflection of the waves by the back wall of the material, and where discontinuities are encountered. The returning signal is captured, mathematically analyzed and presented on a screen, with the resultant waveform showing the location of defects on or within the substrate [7]-[11].

In 2006, Yi-Mei Mao and Pei-Wen Que investigated the possibility of using a then-new sonic signal processing method for inspection of oil pipelines. They compared "ultrasonic signals reflected from defect-free pipelines and from pipelines with defects" [12] and treated the recaptured waveforms with the Hilbert-Huang transform (HHT). The results demonstrated the feasibility of using the technique to successfully locate and determine the extent of discontinuities in oil pipes [12].

Reflected signals are attenuated to different degrees depending on the type of interface they encounter. An interface between a metal and a liquid presents a reduced reflection of the sonic energy, whereas an interface between a gas and a metal causes nearly $100 \%$ reflection of the sonic waves [7]. The actual percentage of reflection is dependent on the ratio of the parameters of certain physical properties between the two types of material, for example, the ratio between the metal and the liquid substance at the interface [13]-[16].

Cracks, holes, laminations, slags, cavities, porosities, bursts, lack of fusion, flakes, lack of penetration and other discontinuities that produce sharp boundaries are easily identified by ultrasonic testing. Other types of discontinuities that produce a more diffuse boundary are still identifiable because they will disrupt the sonic waves in a detectable manner [7] [17].

In 2015, Wissam Alobaidi et al. surveyed seven types of defects commonly found in pipe joint welds, and five often-used types of welds in manufacturing. The correlation between each defect type and the NDT technologies which best reveal the defects is presented in a table [17]. The ability and shortcomings of four NDT techniques commonly used for testing pipe are compared, one of which is ultrasonic testing, and the table reports whether each technique can detect surface, or subsurface flaws. The paper examines ways in which new quality assurance techniques can be incorporated alongside the standard methods in order to overcome the shortcomings of current methods, with the aim to reduce labor costs and increase line output [17].

Because the sensing mode of ultrasonic evaluation is a mechanical process, the frequency range is limited to avoid permanent damage to the targeted objects. Frequencies used most often range from $0.1 \mathrm{MHz}$ to $25 \mathrm{MHz}$. Although Ultrasonic testing (UT) is capable of identifying surface defects, it is primarily used to detect and locate discontinuities that are below the surface, especially in metal parts. UT is useful for other types of inspection, including welds, wall thinning, and surface defects, as mentioned above [1] [7].

This review paper presents applications of, and limitations of some ultrasonic techniques; we will demonstrate the fundamental theory of ultrasound and type of waves used; we will thoroughly examine the inspection approaches of the contact and angle beam techniques. Approaches discussed focus on the measurement of defects in oil and gas pipe manufacturing. Primarily we are interested in determining the depth of the defect $(D D)$ below the material surface. We will also address the limitations of scanning pipe that depend on the transducers used (contact for pipe body and angle beam for welds). Moreover, the paper presents a literature survey for applica- 
tions of ultrasonic techniques in the pipe industry. The primary aim for this review is to investigate possible future coupling of one of the ultrasonic techniques with other NDE techniques, to develop a hybrid detection system for discontinuities. This research study is an accumulation of the practical experience of the authors, as well as the practical experience represented by the reviewed papers.

\section{Fundamental Theory of Ultrasonic}

\subsection{Ultrasound}

Ultrasonic inspection uses sound as the source for testing the medium under consideration. This is the same kind of sound that creates the motion of our eardrums and allows us to hear. The vibrations used for Ultrasonic Testing (UT) are very much higher frequency than what we can hear. But just like any sound wave that moves through the air, the ultrasonic waves that are sent into metal will propagate through the solid medium. When these vibrations encounter interfaces between discontinuous materials (which represent defects in the materials and welds of pipes, for example) they will be reflected in predictable ways. UT is a commonly used method in industries for quality control purposes. It is useful for testing the integrity of metal parts, both before and after forming into pipes. The roll stock can be tested for invisible defects using straight beam ultrasonic testing, allowing the material to be categorized as acceptable, repairable, or scrap before it is incorporated into pipes [7] [17] [18]. Because air does not transmit ultrasound waves as well as solids or gels the difficulty of introducing the signals into metals is overcome by using water or grease as a conducting medium between the transducer and the material to be tested [13].

Ultrasonic testing is used both during the manufacturing of pipe and for inspection of in-service pipelines. The particulars of pipeline insulation may require ingenious ways of getting the ultrasonic transducers into contact with the pipe to be tested. For example, in 2009, H. Lei et al. [19] were interested in developing a device for assessment of the inner walls of underwater oil pipeline. The device, called a pig, uses ultrasonic testing to discover and determine the extent of corrosion within the pipe, while storing the data on a hard disk. Following retrieval of the pig, the recorded data is analyzed in order to determine the reduction in the oil pipe wall, and to identify areas of wall thinning which are then categorized using the American Petroleum Institute (API) standards. The authors claim "perfect performance" of the device [19].

\subsection{Waves}

Waves commonly used are:

- Longitudinal Waves (LW): Another name for these waves is "compression waves". LW is the type of sound wave that we hear, and that is used in manual UT for testing the front end and tail end of the pipe body, and in coil UT for testing the integrity of the plates before they are formed into pipe. The LW pushes the molecules of the tested material in the same direction as the movement of the wave, as shown in Figure 1.

The velocity of the ultrasonic LWs is different in different metals; for example, their velocity through copper is roughly $4760 \mathrm{~m} / \mathrm{s}$, making them the most rapidly propagating ultrasonic waves used in NDT. Through the analysis of wave velocity the depth of defect (DD) can be found [7] [20] [21]. This is what we demonstrated in Section 4.1.

- Shear waves (SW): Shear waves, also known as transverse waves, propagate more slowly and at shorter wavelengths than LWs at equal frequencies. The particle motion is at right angles to the movement of the wave, as shown in Figure 2. SW is usually used for angle beam UT (for example, to detect discontinuities in both the inner diameter and outer diameter of the weld in pipes). As with LW, the SW velocity varies with the type of metal. Some example velocities and corresponding metal types are: Aluminum, roughly $3040 \mathrm{~m} / \mathrm{s}$; Steel, 347 Stainless, roughly $3100 \mathrm{~m} / \mathrm{s}$. When SWs reflect from an interface they sometimes become LWs [7] [20] [22] [23]. This is demonstrated in Section 4.2.

- Rayleigh waves (RW): These waves, which penetrate the material only to the sub-surface distance of one wavelength (at any given frequency), are also called Surface waves. RW travels along the surface of the tested material at velocities equal to those of SWs. RWs are useful for detecting cracks that break the surface of the tested part. They are also useful for testing pieces with intricate rounded surface features. Any defect in zone $\alpha$ as shown in Figure 3, would rest deeper than the wavelength $(\lambda)$ of the test signal, and would likely not be detectable by RW [7] [20] [22]. 


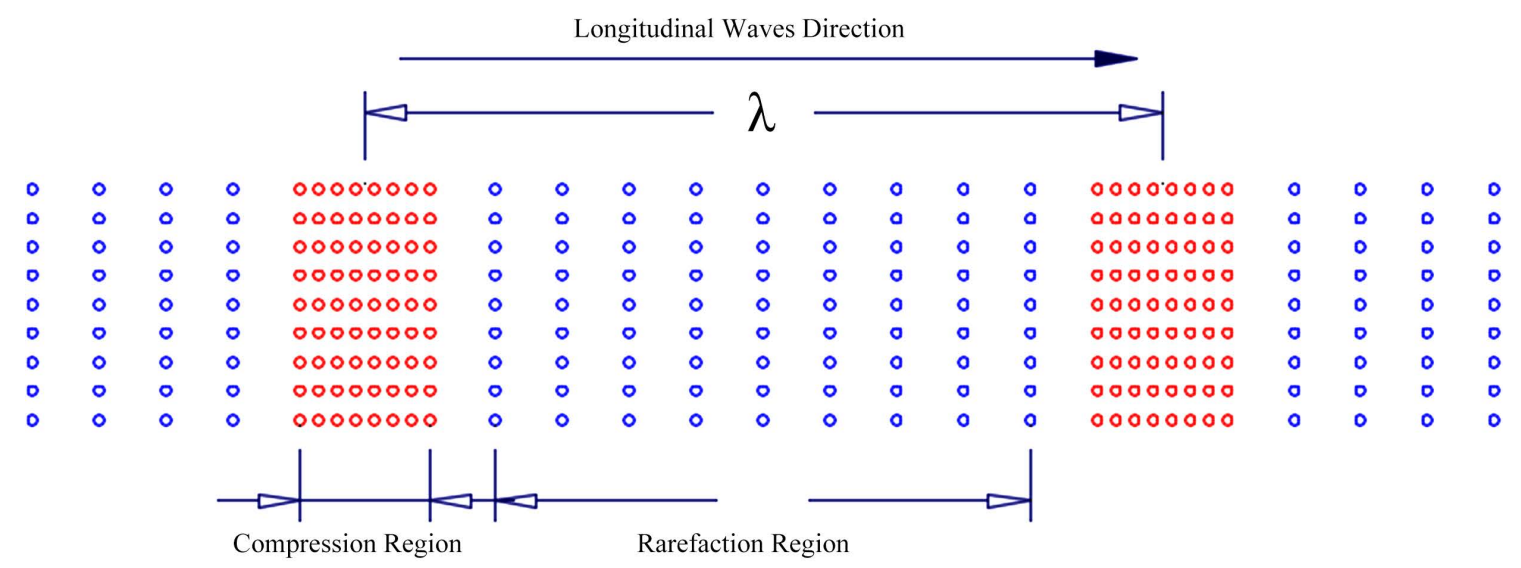

Figure 1. Graphical depiction of parallel motion response of material particles subjected to longitudinal ultrasonic waves, showing compression and rarefaction regions.

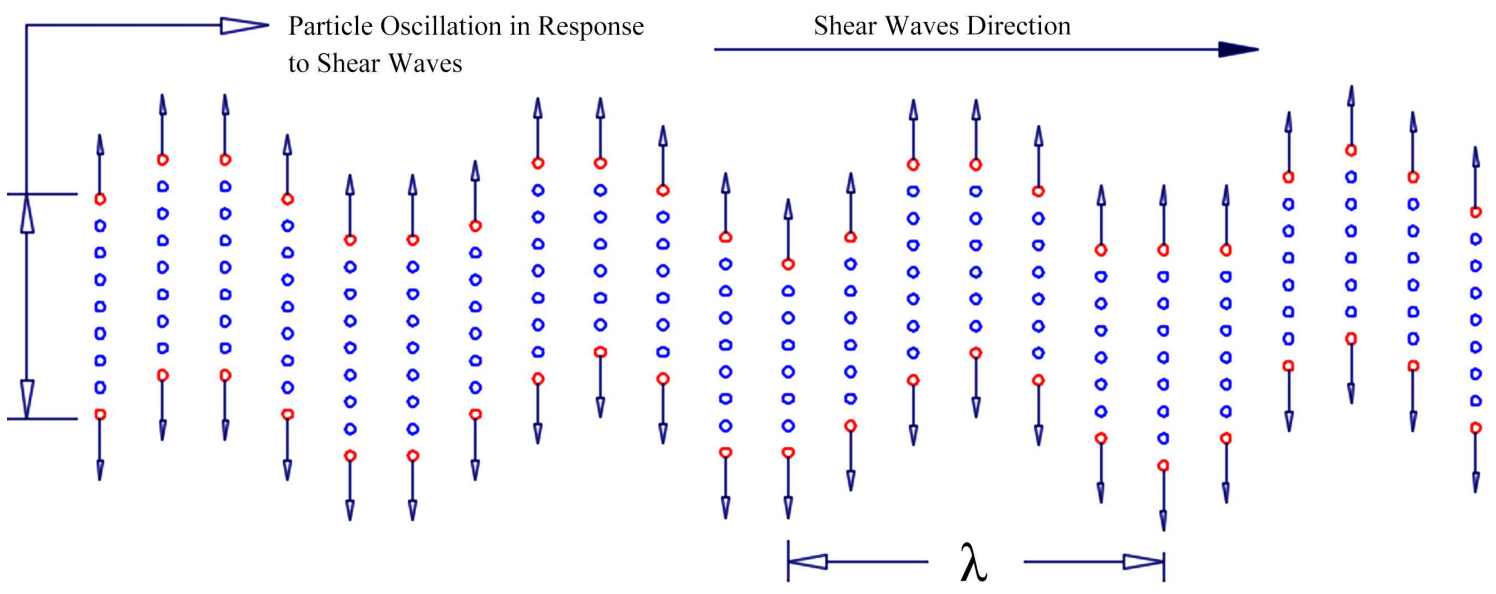

Figure 2. Graphical depiction of perpendicular motion response of material particles subjected to shear ultrasonic waves, showing wavelength.

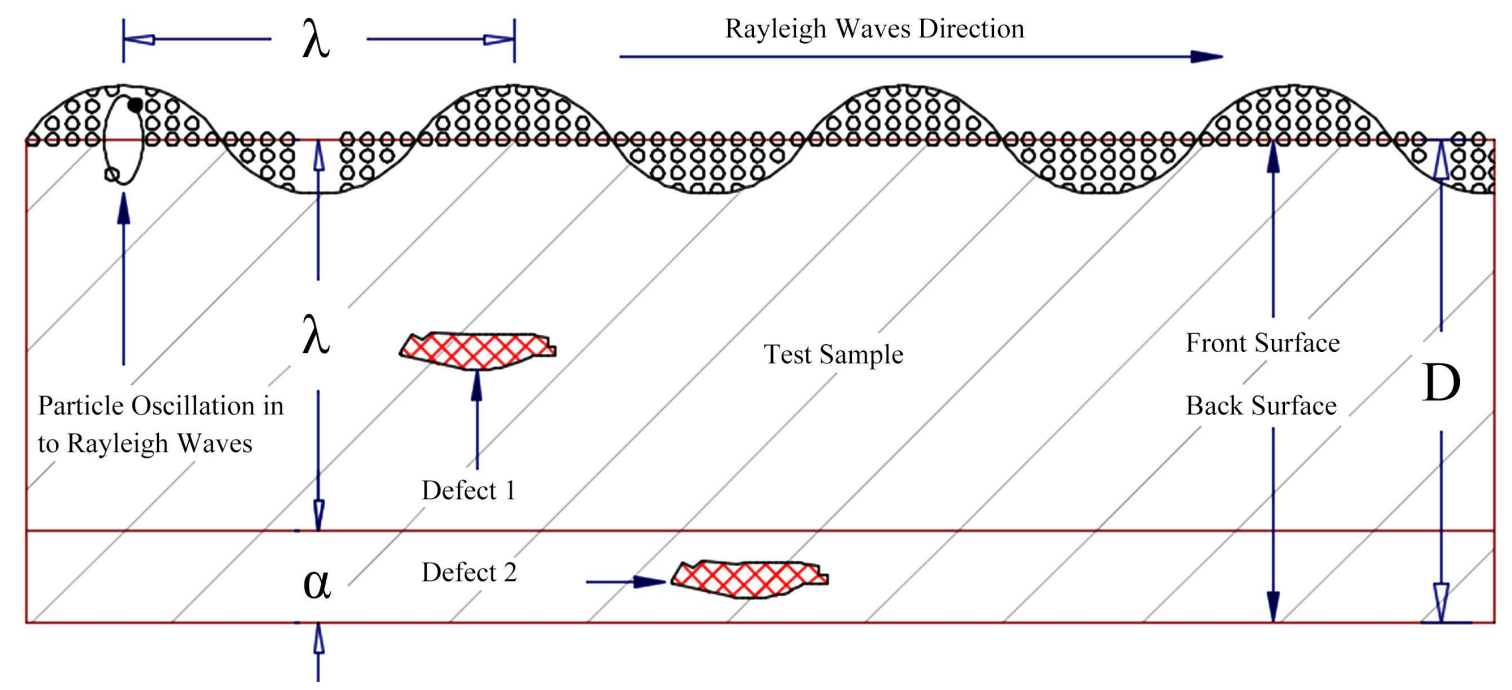

Figure 3. Graphical depiction of limited detection area of Rayleigh waves, showing how they are confined mostly to the surface of a material. 
In 2014, N. P. Aleshin et al. examined various methods and devices for proficiently introducing ultrasonic signals into pipelines with thicknesses ranging between $6 \mathrm{~mm}$ to $20 \mathrm{~mm}$. They covered the choice between Surface and Plate wave modes for conducting these assessments [24].

- Lamb waves (LMW): Lamb waves are vibrations that occur from the upper to the lower surface (up to several wavelengths in thickness) of the tested material, usually a plate (composites or metals), so they are also called Plate waves. They propagate not only through the full thickness of the tested material but are capable of propagating from a single point of excitation over significant distances within the material, as shown in Figure 4. Because the LMWs travel through the solid in a way that is significantly like the behavior of electromagnetic waves within a waveguide, the characteristics of transmission vary from material to material. The velocity of LMWs is dependent on many factors, including density, plate thickness, and the elastic properties of the material being tested [7] [25].

In 2005, Kevin R. Leonard et al. explored use of "helically propagating Lamb waves” transmitted and recaptured with longitudinal transducers. They describe a "meridional-array scheme” [26] used to test the concept of using tomography to detect location and extent of discontinuities in pipes. The research sample used wall-thinning as the defect to be detected. The study also investigated improved reconstruction programs for assessment of helical array signals where "the transmitters and receivers lie along circumferential parallel rings", confirming that frequency compounding reduces noise and artifacts, leading to clearer imagery [26].

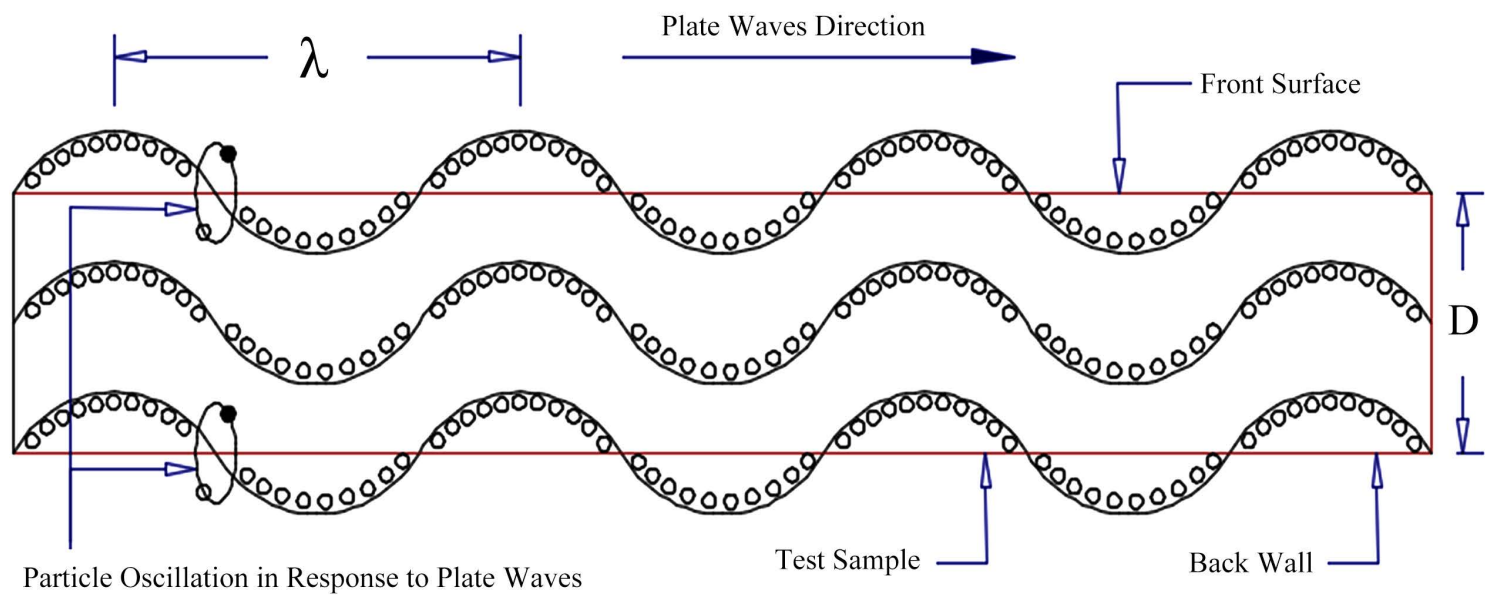

Figure 4. Graphical depiction of ultrasonic lamb waves (plate waves) showing how they move through a test object of a certain thickness which is directly related to the wavelength.

\section{Transducers}

There are five general categories of ultrasonic transducers used in NDE: straight beam, angle beam, dual element, delay line and immersion transducers. Straight beam and angle beam transducers are used in pipe manufacturing NDE procedures. Usually, the standardized inspection codes will determine the type of transducer the manpower (operator) uses for a particular test. In the case where there is no specification or customer requirements, the operator will select a suitable transducer based on prior experience and knowledge [27]-[29]. Many studies investigated ways to reduce the manpower needed for inspection of pipes by using ultrasonic waves, and also ways to reduce the number of transducers required for an inspection protocol, in order to save capital expense.

In 1998, M. J. S. Lowe et al. [30] studied the use of a single transducer for pulse-echo testing of in-service pipeline in order to reveal corrosion in insulated pipes, including oil pipes. They removed only part of the pipe insulation, in order to reduce labor cost. The study focused on selection of the most effective wave modes, and understanding the relationships between the size of a flaw and the signal strength when reflected. The technique was in field trials at the time of publication [30].

In 2004, M. H. S. Siqueira et al. investigated the use of a single transducer to replace groups of transducers that are used for rapidly inspecting pipe. The research assessed the use of guided wave pulse-echo conformations with low ultrasonic signal to noise ratios $(\mathrm{S} / \mathrm{N})$, together with processing via "frequency bandpass filters 
and wavelet analysis” [31]. Their results confirmed the practicality of the concept, showing up to $12 \mathrm{~dB}$ S/N enhancement of the recaptured signal, allowing analysis even with otherwise unusably noisy signals [31].

In 2006, Younho Cho et al. [32] carried out a feasibility study of using guided sonic signals for remote monitoring of stainless steel pipe. They report that their experimental approach, intended to allow them to optimize the guided wave mode resulted in the discovery that "Predicted modes could be successfully generated by controlling frequency, receiver angle and wavelength" [32]. By analyzing scattering patterns mode by mode, they were able to determine that "mode conversion characteristics are distinct depending on dispersive pattern of modes" [32].

\section{Approaches}

\subsection{Straight Beam Evaluation}

The ultrasonic signal used for UT is not continuous. A brief pulse of ultrasound is emitted into the test material from a transducer; the signal travels through the test piece thickness and echoes from either the back wall of the piece, or from a discontinuity within the piece. The echoing signal is captured by the transducer only a few microseconds after being emitted. This gives the process the name pulse-echo [33].

The velocity of travel within the tested material must be known in order to calculate both the presence and the depth of the defect. In a flawless test piece, the distance down and back would each equal $D$, the full thickness of the metal. Thus, the transit time $T$ represents the sonic waves propagating from probes S1 and S2 in both cases in Figure 5 and the reflection from the back wall of the piece, so for the full thickness the total travel will be $2 D$. The factor $\left(V_{L}\right)$ is the velocity of LWs within the type of metal tested, as shown in Equation (1). If the signal finds a defect, the transit time $T_{D}$ would represent the sonic waves propagating from probe S3 in case 2 and the reflection from the interface of the defect, so the distance traveled will be $2 D D$ where $D D$ is the distance from the front surface to the interface with the defect as shown in Figure 5, case 2. Thus, the velocity of LWs $\left(V_{L}\right)$ in this case is used to determine $D D$, and $T_{D}$ is the time of transit to and from the defect, as shown in Equation (2) [34].

$$
\begin{gathered}
V_{L}=\frac{2 D}{T} \\
V_{L}=\frac{2 D D}{T_{D}}
\end{gathered}
$$

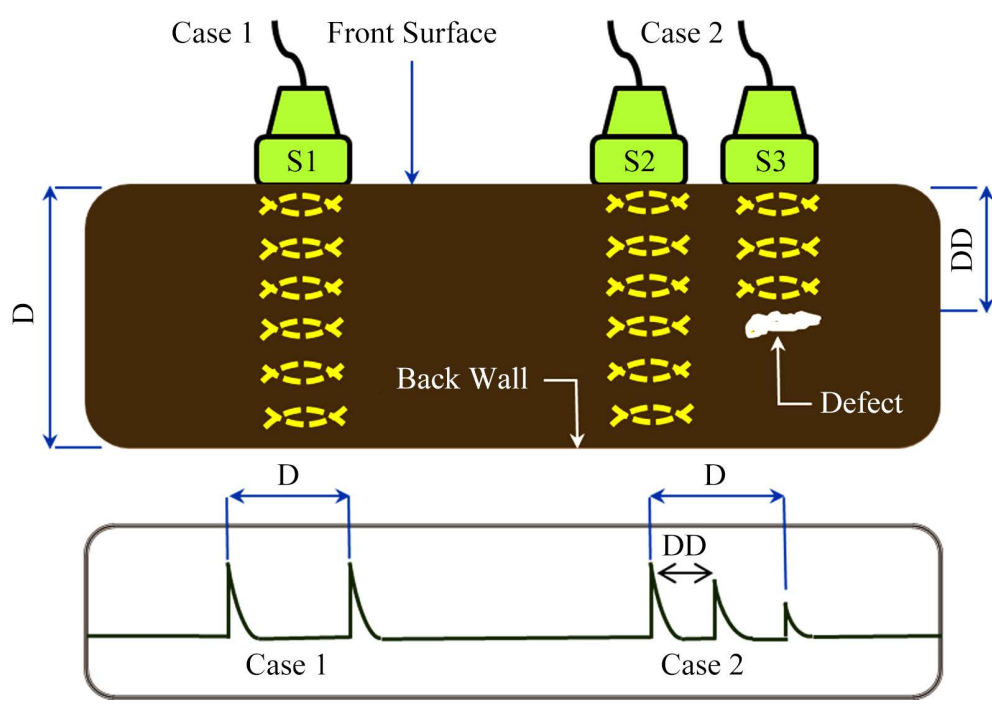

Figure 5. Scheme for the test sample. Two cases are represented. Case 1 has S1 (straight beam \#1). Case 2 has S2 (straight beam \#2) and S3 (straight beam \#3). This figure represents calculation of the value $D D$, using Equation (2). See Section 6. Discussion. 
Straight-beam testing is commonly used in pipe manufacturing to test the roll stock which will be used for building the pipe body. Straight beam testing is effective for detecting cracks that occur parallel to the surfaces of the tested material as well as discontinuities within the body of the material, such as voids or areas of porosity, and inclusions [35].

Figure 6 shows a test sample including eight cases, with the case number shown on the probes from 1 to 8 , respectively. This demonstrates the measurement of the various distances calculated as $D D$ according to Equation (1) and Equation (2) above, and as graphed in Figure 7. The velocities of sonic waves penetrating different materials are compared in Table 1 . These known constant values are necessary to calculate the distance to defect depending on the material from which the tested object is made. The eight cases are calculated for each of six materials, given different transit times for each, as shown in Figure 7, which compares the curves resulting from the eight defects in different materials.

In practical use researchers design mechanisms in order to test their ideas. In 2013, Jin-Sheng Yang et al. [36] reported a system to measure variations in the wall thickness of gas pipe using ultrasound based on the standard cleaning pig with some adaptations. To remove the need of a couplant, the system incorporated a wheel made of an elastic substance, which through mechanical tightness of fit conveyed the vibrations to the pipe wall from a standard piezoelectric probe. The paper covers the operational principle and the system design [36].

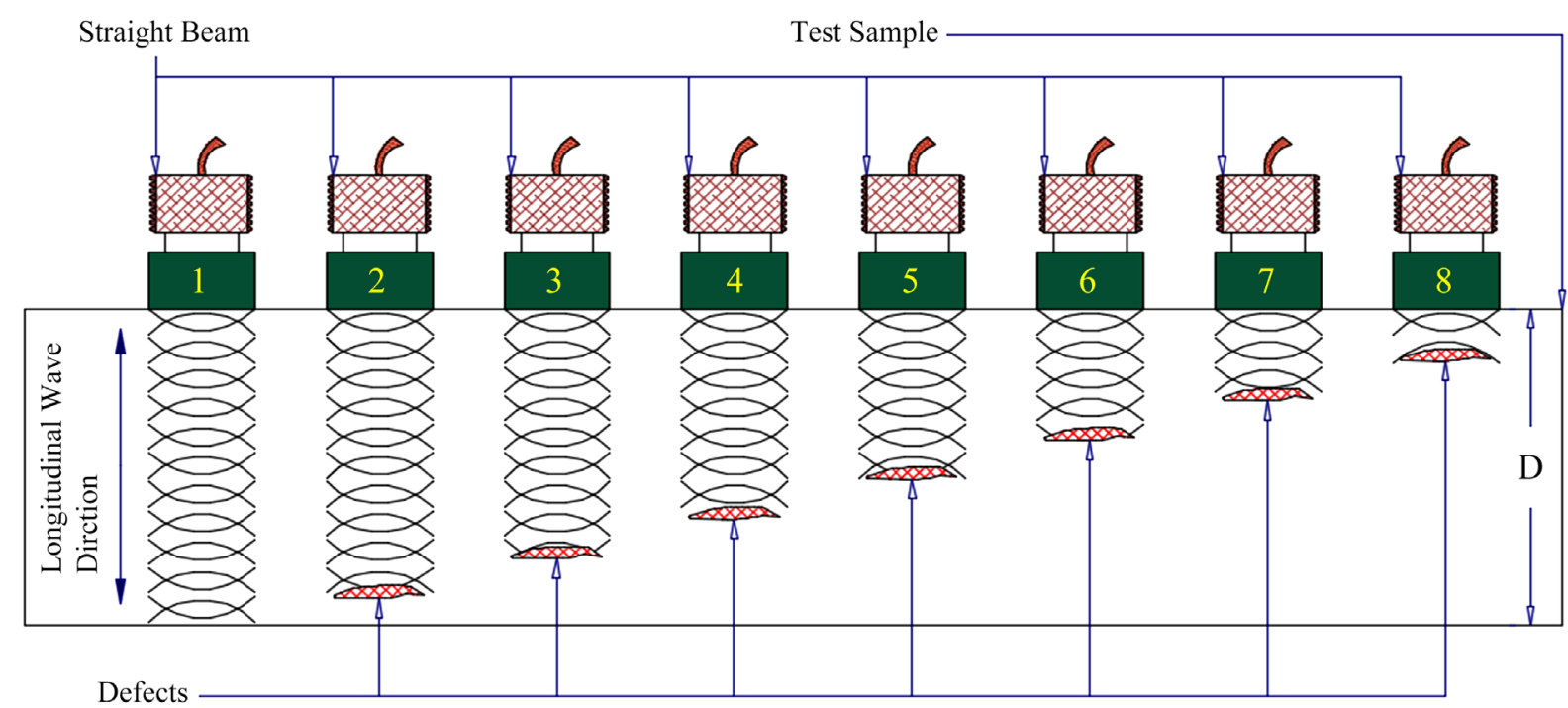

Figure 6. A test sample with 8 cases of defects showing the reflection of test signals from straight beam probes.

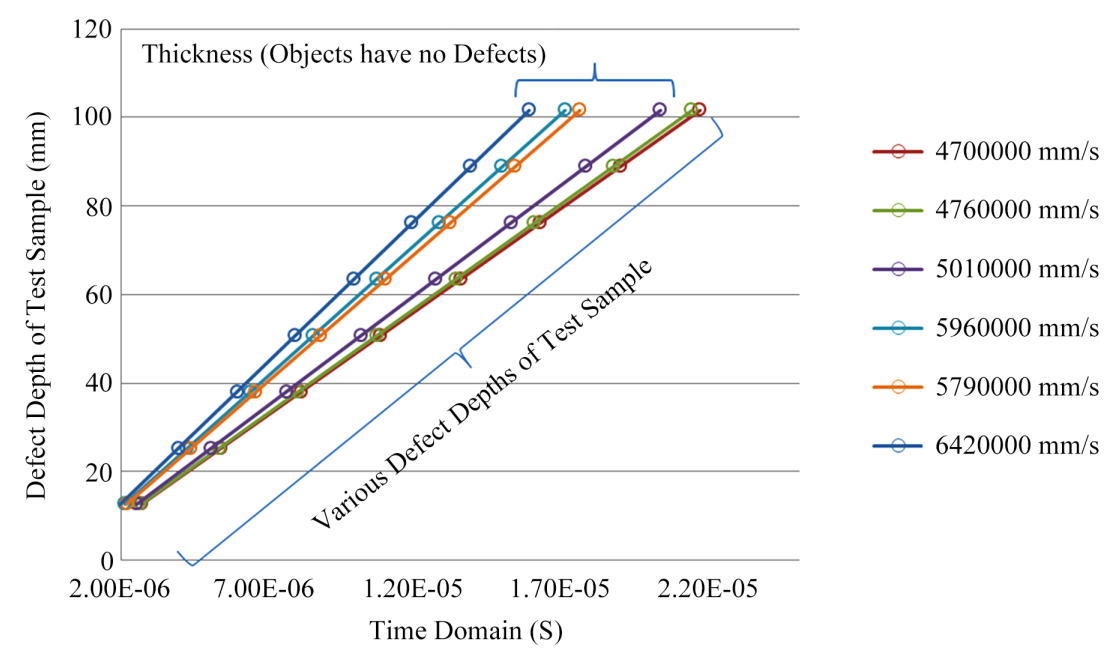

Figure 7. The time domain for the 8 cases from Figure 6 are graphed for each of the 6 materials in Table 1. 
Table 1. Longitudinal wave velocities shown for 6 different materials.

\begin{tabular}{ccccccc}
\hline Materials & $\begin{array}{c}\text { Aluminum, } \\
\text { rolled }\end{array}$ & Steel, mild & $\begin{array}{c}\text { Steel, } 347 \\
\text { stainless }\end{array}$ & Copper, rolled & $\begin{array}{c}\text { Copper, } \\
\text { annealed }\end{array}$ & Brass \\
\hline$V_{L}(\mathrm{~mm} / \mathrm{s})$ & $6,420,000$ & $5,960,000$ & $5,790,000$ & $5,010,000$ & $4,760,000$ & $4,700,000$ \\
\hline
\end{tabular}

\subsection{Angle Beam Evaluation}

Straight beam ultrasonic techniques are best for locating defects in plate-type materials where defects are often parallel to the surface of the object, but they are ineffective for testing welds. The discontinuities in welds are usually at an angle to the surface of the sample under test. Beams approaching the weld interface at an angle are effective at detecting discontinuities within the welding bead. The angle beam transducer is used to generate the test signal in the majority of ultrasonic inspections. To assess discontinuities using angle beam examination, skip distance (SKD) is used to describe the sound path (SP) reflected from the back wall interface (1st leg) and going immediately to reflecting, where it is again reflected from the front wall (2nd leg). SKD is formed by the full SP (1st leg and 2nd leg), and is the distance from the point of excitation to the end of the second leg, as shown in Figure 8 [17] [37]-[39].

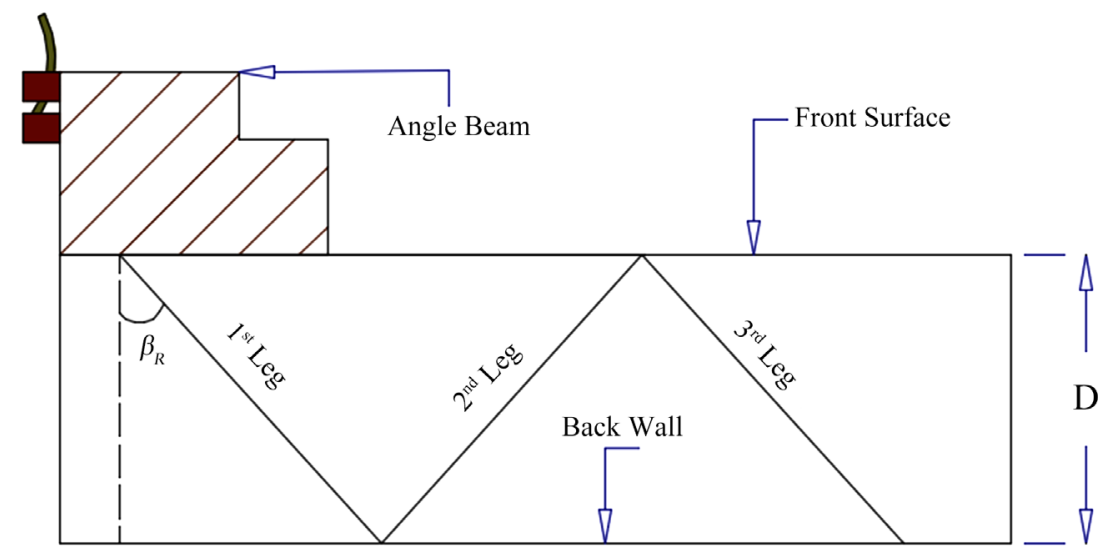

Figure 8. Representation of skip distance through three legs, $1 / 2$ skip, full skip and $1 \frac{1 / 2}{2}$ skip. $D$ is the material thickness and $\beta_{R}$ SP angle.

All the equations below are adapted from [37]-[39].

$$
\operatorname{SKD}=2 D \operatorname{Tan}\left(\beta_{R}\right)
$$

Surface distance (SD) is equal to half SKD.

$$
\mathrm{SD}=D \operatorname{Tan}\left(\beta_{R}\right)
$$

These calculations cannot be completed unless $D$ and $\beta_{R}$ to the front surface are known. If the length of the 1st leg is known, the SKD and SD can be computed as.

$$
\operatorname{SKD}=2(L 1) \operatorname{Sin}\left(\beta_{R}\right)
$$

and

$$
\mathrm{SD}=\operatorname{L1Sin}\left(\beta_{R}\right)
$$

The segments of the SP numbered $L 1, L 2$ and $L 3$ in Figure 8 are 1st leg (L1), 2nd leg (L2) and 3rd leg (L3) respectively. And through the trigonometric functions SKD, SD, $L 1, L 2$ and $L 3$ can be calculated as shown in Table 2, by taking 70, 65, 60, 55, 50 and 45 degrees as the $\beta_{R}$. The first leg and second leg are calculated as follows. 


$$
L 1=\frac{D}{\operatorname{Cos}\left(\beta_{R}\right)}
$$

and

$$
L 1+L 2=\frac{2 D}{\operatorname{Cos}\left(\beta_{R}\right)}
$$

According to the table below, we graphed the SP angle against the SKD. These graphs show the limitations of the scan distance to the pipe weld depending on $\beta_{R}$. Where, SKD and SP length depends on the propagating sonic angle. That means, the larger the angle is, the greater the scanning distance becomes, based on the ( $1 / 2$, Full and 1 1/2) for SKD and SP as shown in Figure 9 and Figure 10.

To calculate the depth of the discontinuity $(D D)$, we measure vertically from the point of reflection at the interface with the defect, up to the front surface of the test sample [34], as shown in Figure 11 and Figure 12. To calculate the $D D$ 's, the values of $D$, SKD and SD must be known, as shown below. Figure 11 shows a defect interrupting the path of $L 1$. Figure 12 shows a defect interrupting the path of $L 2$.

$$
\begin{gathered}
D D=L 1 \operatorname{Cos}\left(\beta_{R}\right) \\
D D=2 D-\left[\left(\operatorname{Cos} \beta_{R}\right)(L 1+L 2)\right]
\end{gathered}
$$

Table 2. Summary and comparison of the SKD and SP lengths for 6 different beam angles.

\begin{tabular}{cccccccc}
\hline \multirow{2}{*}{$\beta_{R}$} & SD $(\mathrm{mm})$ & \multicolumn{3}{c}{ SKD $(\mathrm{mm})$} & \multicolumn{3}{c}{ SP $(\mathrm{mm})$} \\
\cline { 3 - 8 } & & $1 / 2 \mathrm{SKD}$ & Full SKD & $1(1 / 2)$ SKD & $1 / 2$ SP & Full SP & $1\left(\frac{1}{2}\right)$ SP \\
\hline 45 & 25.4 & 25.4 & 50.8 & 76.2 & 36 & 72 & 108 \\
50 & 30.27 & 30.27 & 60.54 & 90.81 & 39.515 & 79.03 & 118.545 \\
55 & 36.275 & 36.275 & 72.55 & 108.825 & 44.3 & 88.6 & 132.9 \\
60 & 44 & 44 & 88 & 132 & 50.8 & 101.6 & 152.4 \\
65 & 54.47 & 54.47 & 108.94 & 163.411 & 60.1 & 120.2 & 180.3 \\
70 & 69.786 & 69.786 & 139.572 & 209.358 & 74.265 & 148.53 & 222.8 \\
\hline
\end{tabular}

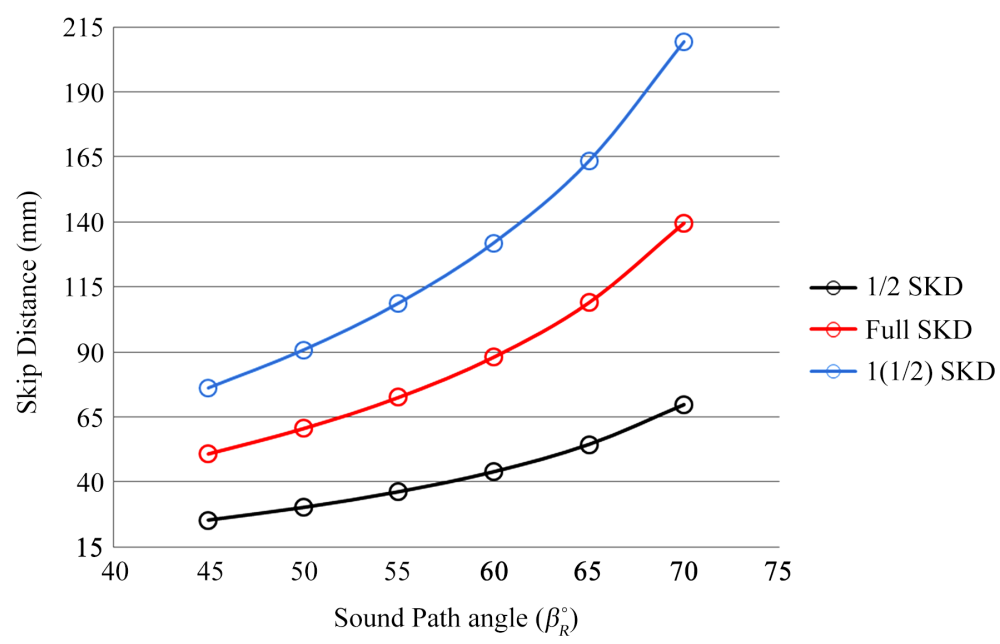

Figure 9. Skip distance plotted against sound path (SP) angle, showing the SP distances achieved with $1 / 2$, full and $1 \frac{1 / 2}{2}$ SKD, for use by the operator in selecting the SP angle to be used during test depending on the material. 


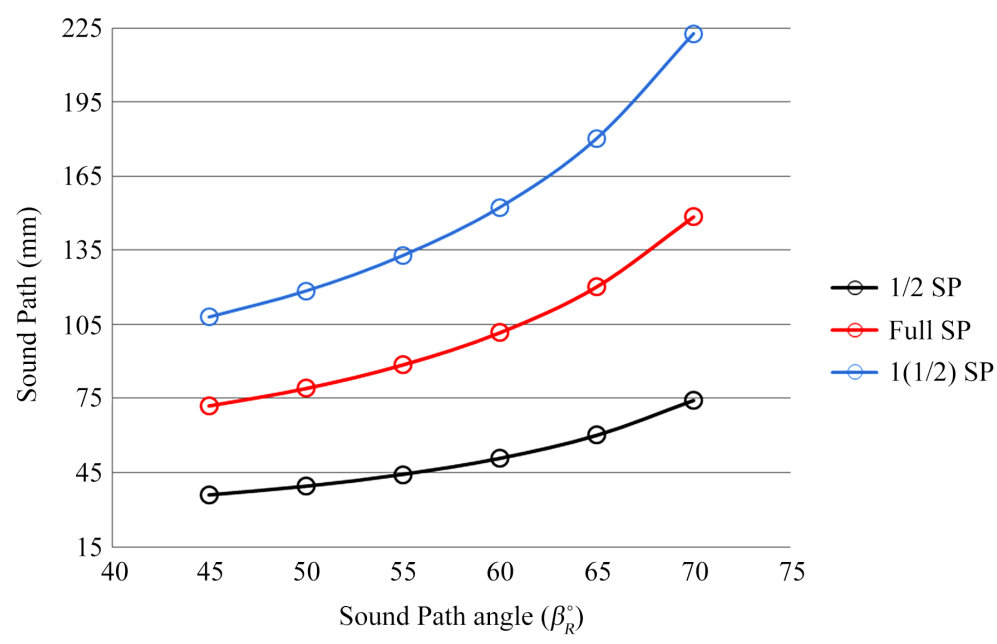

Figure 10. Sound path length plotted against SP angle. This shows the exact length of penetration of sonic waves according to the material for $1 / 2$, full and $1 \frac{1}{2}$ SKD.

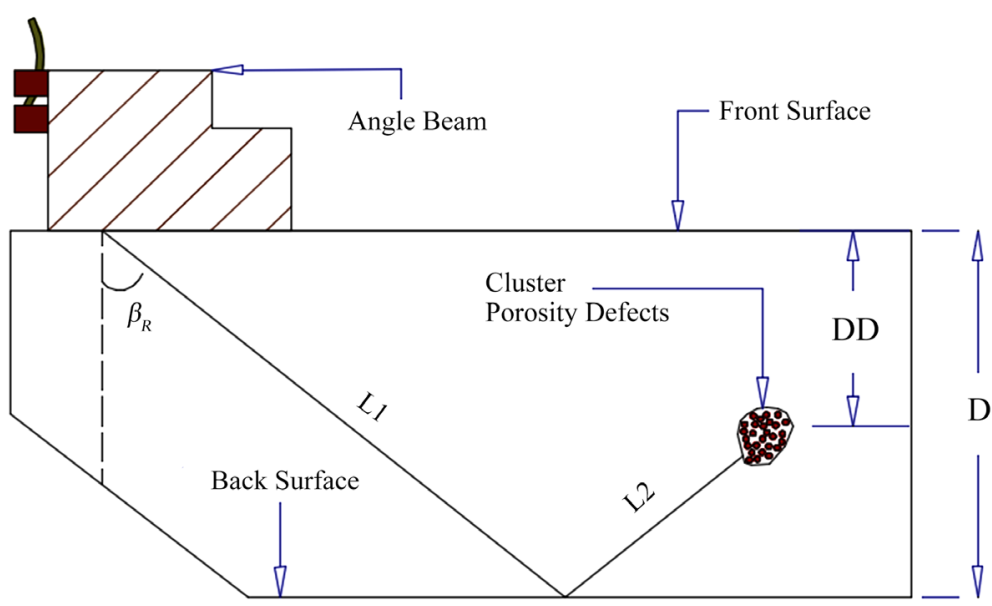

Figure 11. Illustration of the test object and defect depth found by angle beam transducer (defect found by the 2 nd leg).

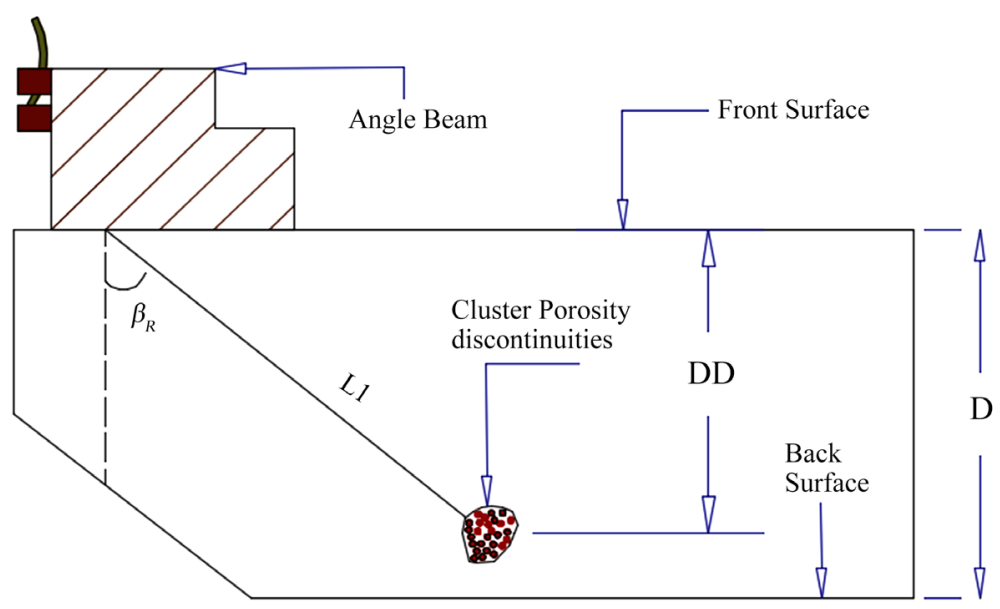

Figure 12. Illustration of the test sample and discontinuity depth as located by angle beam transducer (defect located by the 1st leg). 
Figure 13 shows a schematic of a pipe cross section containing a cluster porosity discontinuity in the pipe weld. This is an example of one type of discontinuity that is best discovered by using an Angle Beam Transducer.

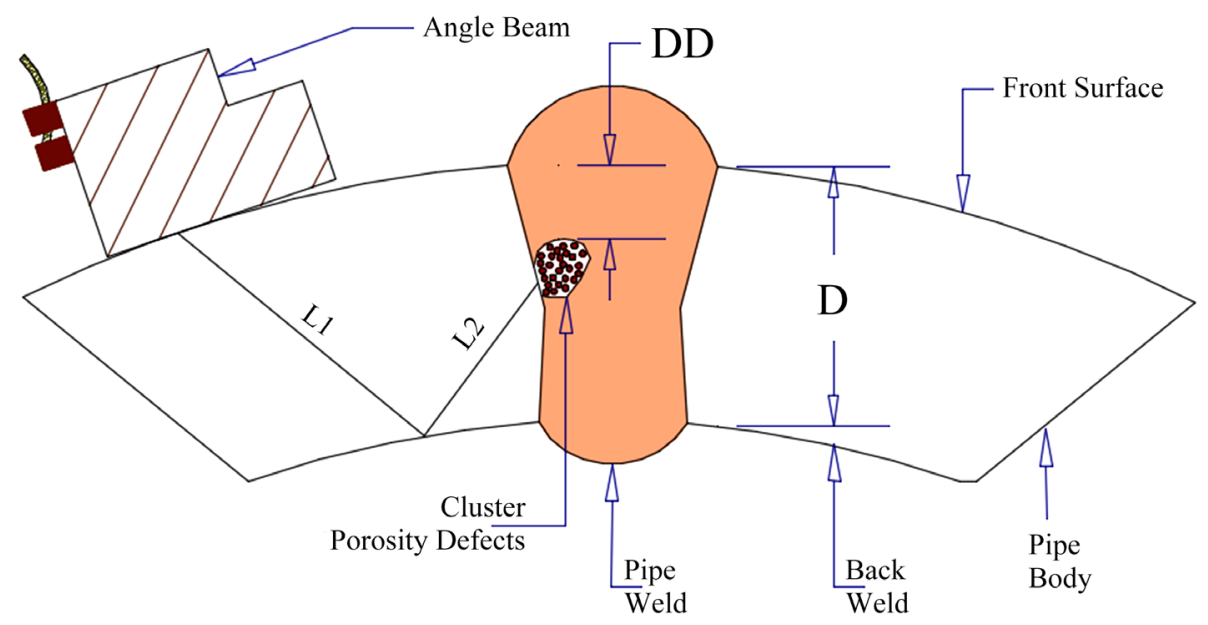

Figure 13. Pipe cross section schematic of discontinuity depth discovered on the 2nd leg by angle beam transducer.

\section{Summary}

Ten analyses done by ultrasonic NDE specialists are listed in Table 3 below which summarizes the problems studied by each paper, the name of the first author and the year of publication.

Table 3. Selected prior research into uses of ultrasonic NDT to detect discontinuities in pipeline materials.

\begin{tabular}{|c|c|c|c|}
\hline No. & Problems & First author name & Year \\
\hline 1 & Defect detection in pipes using guided waves & M. J. S. Lowe & 1998 \\
\hline 2 & The use of ultrasonic guided waves and wavelets analysis in pipe inspection & M. H. S. Siqueira & 2004 \\
\hline 3 & Lamb wave tomography of pipe-like structures & Kevin R. Leonard & 2005 \\
\hline 4 & $\begin{array}{l}\text { A wall thinning detection and quantification based on guided wave } \\
\text { mode conversion features }\end{array}$ & Younho Cho & 2006 \\
\hline 5 & $\begin{array}{l}\text { Application of Hilbert-Huang signal processing to ultrasonic } \\
\text { non-destructive testing of oil pipelines }\end{array}$ & Yi-Mei Mao & 2006 \\
\hline 6 & Ultrasonic phased array inspection of electrofusion joints in polyethylene pipes & D. S. Caravaca & 2007 \\
\hline 7 & Ultrasonic pig for submarine oil pipeline corrosion inspection & H. Lei & 2009 \\
\hline 8 & A new type of wheeled intelligent ultrasonic thickness measurement system & Jin-Sheng Yang & 2013 \\
\hline 9 & Automatic ultrasonic inspection of large-diameter pipes & N. P. Aleshin & 2014 \\
\hline 10 & A survey on benchmark defects encountered in the oil pipe industries & Wissam Alobaidi & 2015 \\
\hline
\end{tabular}

\section{Discussion}

To summarize the objectives of our research reported in this paper are?

- Extending the service life of pipes through early quality control testing by finding more effective and appropriate ways to evaluate them.

- Demonstrating the limitations of two Ultrasonic testing techniques as covered in sections above.

- Employing the technique to extend the effectiveness of this technology in determining the location and depth of defects. 
- Coupling ultrasonic technology with another NDT technique in an automated system to quickly determine the exact location and degree of the discontinuity.

- After designing a new system merging the Ultrasonic technique with another NDT technique, to build the actual system and employ it in real-world conditions to remotely detect and evaluate discontinuities.

The focus here will be on approaching straight beam and angle beam testing, with each considered as a different technique. The first is a longitudinal wave mode where the wave direction is parallel to the SP, and the second one is a shear wave mode where the wave direction is perpendicular to the SP. These techniques have different uses, as the straight beam is best for the pipe body and the angle beam for the pipe weld. As the review shows, the limitations for these techniques have been examined before. It should be mentioned that, while preparing a newly designed system, the limitations and advantages of the techniques must be considered. The resulting new system must be fit and appropriately in line with these technical conditions. As shown in Figure 5, the straight beam probe transmits an ultrasonic wave into the test object. In Case 1 there is no defect, thus the beam travels the full thickness of the material. The peaks for Case 1 represent the origination of the sonic signal, and the reflection of the signal from the back wall. In Case 2 probe S2 sends an unimpeded signal, which behaves like that in Case 1. But S3 sends a signal which is reflected by a defect, so the signal reflection arrives in less time, represented by the middle peak in the graph for Case 2. This yields the distance to defect $(D D)$.

A consideration for angle beam testing is that the attenuation of the sonic waves varies depending on the material from which the object is made. As shown in Figure 9 and Figure 10, $\beta_{R}$ is adjusted according to the degree of penetration possible with a given material. For a more penetrable substance, $\beta_{R}$ is greater, allowing a greater SKD, while a less penetrable substance requires a smaller $\beta_{R}$, with a respective reduction in SKD.

\section{Conclusions}

It is usual practice to base an NDT research on the various materials used in order to estimate and evaluate the suitability of ultrasonic NDE for testing the materials involved. The next step is to develop the needed devices, then to investigate the problem of using the ultrasonic NDE technique to assess the structural objects.

Through previous research, and the techniques that have been focused on, it can be seen that the straight probe works up to a limited thickness (such as the thickness of a pipe wall), and is employed to reveal subsurface defects, especially cracks that are parallel to the front and back surfaces of the test object. The example given in this article demonstrates a pipe wall with $D=50.8 \mathrm{~mm}$, with eight cases of defect where $D D$ ranges from $6.35 \mathrm{~mm}$ to $44.45 \mathrm{~mm}$ and presents the graphed results based on six different materials commonly used in the pipe construction industry (shown in Figure 7).

The angle beam is used to reveal discontinuities in the weld regions, with the limitation that the SKD cannot be increased unless the SP angle is increased. And that increased distance attenuates the sonic energy. The examples included in this article calculated the limitations of effective SD, SKD and SP using six reflection angles. Focusing on the $45^{\circ}$ and $70^{\circ}$ angles at the extremes for our example of $D=25.4 \mathrm{~mm}$, we show that the $45^{\circ}$ angle yields a $1 \frac{2}{2}$ SKD $(25.4 \mathrm{~mm})$, full SKD $(50.8 \mathrm{~mm})$ and $1 \frac{1 / 2}{2} \operatorname{SKD}(76.2 \mathrm{~mm})$ with higher energy if we compare to the same material but a different angle, such as $70^{\circ}$ angle which a greater test distance, but with more signal attenuation. Using the $70^{\circ}$ angle we get $1 / 2$ SKD $(69.786 \mathrm{~mm})$, full SKD $(139.572 \mathrm{~mm})$, and $1 \frac{1}{2} \mathrm{SKD}$ (209.358 $\mathrm{mm}$ ). The greater reach leads to reduced sensitivity because of the signal attenuation; operators must balance these factors when ultrasonic NDE is used. The general understanding is that $1 \frac{1 / 2}{2} \mathrm{SKD}$ is the accepted limit of successful range for angle beam testing, all that according to the previous studies and technical websites. This requires continual attention by customer requirement and if there is no requirement, by the operator when assessing the integrity of pipes during manufacture.

It is recommended that a second, powerful NDE technique capable of remote detection would be useful alongside ultrasonic testing in order to direct the placement of the probe.

\section{References}

[1] NDT Resource Center, The Collaboration for NDT Education, Iowa State University (2001-2014) Introduction to Ultrasonic Testing (Basic Principles of Ultrasonic Testing). https://www.nde-ed.org/EducationResources/CommunityCollege/Ultrasonics/Introduction/description.htm

[2] Beard, M.D. and Lowe, M.J.S. (2003) Non-Destructive Testing of Rock Bolts Using Guided Ultrasonic Waves. International Journal of Rock Mechanics \& Mining Sciences, 40, 527-536. 
http://dx.doi.org/10.1016/S1365-1609(03)00027-3

[3] Chaki, S. and Bourse, G. (2009) Guided Ultrasonic Waves for Non-Destructive Monitoring of the Stress Levels in Prestressed Steel Strands. Ultrasonics, 49, 162-171. http://dx.doi.org/10.1016/j.ultras.2008.07.009

[4] Drinkwater, B.W. and Wilcox, P.D. (2006) Ultrasonic Arrays for Non-Destructive Evaluation: A Review. NDT \& E International, 39, 525-541. http://dx.doi.org/10.1016/j.ndteint.2006.03.006

[5] Dutton, B., Clough, A.R., Rosli, M.H. and Edwards, R.S. (2011) Non-Contact Ultrasonic Detection of Angled Surface Defects. NDT \& E International, 44, 353-360. http://dx.doi.org/10.1016/j.ndteint.2011.02.001

[6] Caravaca, D.S., Bird, C. and Kleiner, D. (2007) Ultrasonic Phased Array Inspection of Electrofusion Joints in Polyethylene Pipes. Insight: Non-Destructive Testing and Condition Monitoring, 49, 83-86.

http://dx.doi.org/10.1784/insi.2007.49.2.83

[7] Davis, J.R. (1992) Nondestructive Evaluation and Quality Control. ASME Handbook, 17, 486-494.

[8] Matz, V., Kreidl, M. and Šmíd, R. (2005) Classification of Ultrasonic Signals. The 8th International Conference of the Slovenian Society for Non-Destructive Testing. Application of Contemporary Non-Destructive Testing in Engineering, Portoroz, 27-33.

[9] Mazeika, L., Kazys, R., Raisutis, R. and Sliteris, R. (2011) Ultrasonic Guided Wave Tomography for the Inspection of the Fuel Tanks Floor. International Journal of Materials and Product Technology, 41, 128-139. http://dx.doi.org/10.1504/IJMPT.2011.040291

[10] McNab, A. and Young, H.S. (1989) Knowledge-Based Approach to the Formulation of Ultrasonic Nondestructive Testing Procedures. IEE Proceedings, Science, 136, 134-140.

[11] Nandi, A.K., Mampel, D. and Roscher, B. (1997) Blind Deconvolution of Ultrasonic Signals in Nondestructive Testing Applications. IEEE Transactions on Signal Processing, 45, 1382-1390. http://dx.doi.org/10.1109/78.575716

[12] Mao, Y. and Que, P. (2006) Application of Hilbert-Huang Signal Processing to Ultrasonic Non-Destructive Testing of Oil Pipelines. Journal of Zhejiang University SCIENCE A, 7, 130-134. http://dx.doi.org/10.1631/jzus.2006.A0130

[13] Ricci, M., Senni, L. and Burrascano, P. (2012) Exploiting Pseudorandom Sequences to Enhance Noise Immunity for Air-Coupled Ultrasonic Nondestructive Testing. IEEE Transactions on Instrumentation and Measurement, 61, 29052915. http://dx.doi.org/10.1109/TIM.2012.2200409

[14] Salazar, A.J. and Rodríguez, E.C. (2011) Studies of the Effect of Surface Roughness in the Behaviour of Ultrasonic Signals in AISI-SAE-4340 Steel: Spectral and Wavelets Analysis. International Journal of Microstructure and Materials Properties, 6, 224-235. http://dx.doi.org/10.1504/IJMMP.2011.043218

[15] Silva, C.E.R., Alvarenga, A.V. and Costa-Felix, R.P.B. (2012) Nondestructive Testing Ultrasonic Immersion Probe Assessment and Uncertainty Evaluation According to EN 12668-2:2010. IEEE Transactions on Ultrasonics, Ferroelectrics, and Frequency Control, 59, 2338-2346. http://dx.doi.org/10.1109/TUFFC.2012.2459

[16] Zahran, O., Shihab, S. and Al-Nuaimy, W. (2002) Recent Developments in Ultrasonic Techniques for Rail-Track Inspection. Northampton British Institute of Non-Destructive Testing, Brownlow Hill, 55-60.

[17] Alobaidi, W., Sandgren, E. and Al-Rizzo, H. (2015) A Survey on Benchmark Defects Encountered in the Oil Pipe Industries. International Journal of Scientific \& Engineering Research, 6, 844-853.

[18] OLYMPUS Your Vision, Our Future (2015) Ultrasonic Flaw Detection Tutorial (What Is Ultrasound?). http://www.olympus-ims.com/en/ndt-tutorials/flaw-detection/ultrasound/

[19] Lei, H., Huang, Z., Liang, W., Mao, Y. and Que, P.W. (2009) Ultrasonic Pig for Submarine Oil Pipeline Corrosion Inspection. Russian Journal of Nondestructive Testing, 45, 285-291. http://dx.doi.org/10.1134/S106183090904010X

[20] OLYMPUS Your Vision, Our Future (2015) Ultrasonic Flaw Detection Tutorial (Wave Propagation). http://www.olympus-ims.com/en/ndt-tutorials/flaw-detection/wave-propagation/

[21] Lucassen, J. and Tempel, M. (1972) Longitudinal Waves on Visco-Elastic Surfaces. Journal of Colloid and Interface Science, 41, 491-498. http://dx.doi.org/10.1016/0021-9797(72)90373-6

[22] Gabriels, P., Snieder, R. and Nolet, G. (1987) In Situ Measurements of Shear-Wave Velocity in Sediments with Higher-Mode Rayleigh Waves. Geophysical Prospecting, 35, 187-196. http://dx.doi.org/10.1111/j.1365-2478.1987.tb00812.x

[23] Lysmer, J. and Waas, G. (1972) Shear Waves in Plane Infinite Structures. Journal of the Engineering Mechanics Division, 98, 85-105.

[24] Aleshin, N.P., Gobov, Yu.L., Mikhailov, A.V., Smorodinskii, Ya.G. and Syrkin, M.M. (2014) Automatic Ultrasonic Inspection of Large Diameter Pipes. Russian Journal of Nondestructive Testing, 50, 133-140. http://dx.doi.org/10.1134/S1061830914030024

[25] Alleyne, D.N. and Cawley, P. (1992) The Interaction of Lamb Waves with Defects. IEEE Transactions on Ultrasonics, 
Ferroelectrics, and Frequency Control, 39, 381-397. http://dx.doi.org/10.1109/58.143172

[26] Leonard, K.R. and Hinders, M.K. (2005) Lamb Wave Tomography of Pipe-Like Structures. Ultrasonics, 43, 574-583. http://dx.doi.org/10.1016/j.ultras.2004.12.006

[27] OLYMPUS Your Vision, Our Future (2015) Ultrasonic Flaw Detection Tutorial (General Concepts of Transducer Selection). http://www.olympus-ims.com/en/ndt-tutorials/flaw-detection/general-concepts/

[28] NDT Resource Center, The Collaboration for NDT Education, Iowa State University (2001-2014) Introduction to Ultrasonic Testing (Transducer Types). https://www.nde-ed.org/EducationResources/CommunityCollege/Ultrasonics/EquipmentTrans/transducertypes.htm

[29] Lach, M., Platte, M. and Ries, A. (1996) Piezoelectric Materials for Ultrasonic Probes. NDT Net, 1, No. 9. http://www.ndt.net/article/platte2/platte2.htm

[30] Lowe, M.J.S., Alleyne, D.N. and Cawley, P. (1998) Defect Detection in Pipes Using Guided Waves. Ultrasonics, 36, 147-154. http://dx.doi.org/10.1016/S0041-624X(97)00038-3

[31] Siqueira, M.H.S., Gatts, C.E.N., Da Silva, R.R. and Rebello, J.M.A. (2004) The Use of Ultrasonic Guided Waves and Wavelets Analysis in Pipe Inspection. Ultrasonics, 41, 785-797. http://dx.doi.org/10.1016/j.ultras.2004.02.013

[32] Cho, Y., Oh, W.D. and Lee, J.H. (2006) A Wall Thinning Detection and Quantification Based on Guided Wave Mode Conversion Features. Key Engineering Materials, 321-323, 795-798. http://dx.doi.org/10.4028/www.scientific.net/KEM.321-323.795

[33] NDT Resource Center, The Collaboration for NDT Education, Iowa State University (2001-2014) Introduction to Ultrasonic Testing (Normal Beam Inspection). https://www.nde-ed.org/EducationResources/CommunityCollege/Ultrasonics/MeasurementTech/beaminspection.htm

[34] Cleveland, D., Barron, A.R. and Mucciardi, A.N. (1980) Methods for Determining the Depth of Near-Surface Defects. Journal of Nondestructive Evaluation, 1, 21-36. http://dx.doi.org/10.1007/BF00566229

[35] OLYMPUS Your Vision, Our Future (2015) Ultrasonic Flaw Detection Tutorial, Straight Beam Tests (Plates, Bars, Forgings, Castings, etc.). http://www.olympus-ims.com/en/ndt-tutorials/flaw-detection/straight-beam-tests/

[36] Yang, J.-S., Zhao, X.-G., Zhang, Y.-J. and Cao, Z. (2013) A New Type of Wheeled Intelligent Ultrasonic Thickness Measurement System. Symposium on Piezoelectricity, Acoustic Waves and Device Applications (SPAWDA), Changsha, 25-27 October 2013, 1-4. http://dx.doi.org/10.1109/spawda.2013.6841115

[37] NDT Resource Center, The Collaboration for NDT Education, Iowa State University (2001-2014) Introduction to Ultrasonic Testing (Angle Beams I). https://www.nde-ed.org/EducationResources/CommunityCollege/Ultrasonics/MeasurementTech/anglebeam1.htm

[38] NDT Resource Center, The Collaboration for NDT Education, Iowa State University (2001-2014) Introduction to Ultrasonic Testing (Angle Beams II).

https://www.nde-ed.org/EducationResources/CommunityCollege/Ultrasonics/MeasurementTech/anglebeam2.htm

[39] NDT Resource Center, The Collaboration for NDT Education, Iowa State University (2001-2014) Introduction to Ultrasonic Testing (Angle Beam Inspection Calculations). https://www.nde-ed.org/GeneralResources/Formula/AngleBeamFormula/AngleBeamTrig.htm 